\title{
Can the minimum rule of possibility theory be extended to belief functions?
}

\author{
Sébastien Destercke and Didier Dubois \\ Institut de Recherche en Informatique de Toulouse (IRIT), \\ 118 Route de Narbonne, 31400 Toulouse. \\ \{Destercke,Dubois\}@irit.fr
}

\begin{abstract}
When merging belief functions, Dempster rule of combination is justified only when sources can be considered as independent. When dependencies are ill-known, it is usual to ask the merging operation to satisfy the property of idempotence, as this property ensures a cautious behaviour in the face of dependent sources. There are different strategies to find such rules for belief functions. One strategy is to rely on idempotent rules used in either more general or more specific frameworks and to respectively study their particularisation or extension to belief functions. In this paper, we try to extend the minimum rule of possibility theory to belief functions. We show that such an extension is not always possible, unless we accept the idea that the result of the fusion process can be a family of belief functions.
\end{abstract}

keywords: belief functions, idempotence, fusion, possibility

\section{Introduction}

There exist many fusion rules in the theory of belief functions [22], the best known being Dempster's rule [4], either normalized or not. When several sources deliver information over a common frame of discernment $\mathcal{X}$, combining belief functions by Dempster's rule is justified only when the sources can be assumed to be independent. However, such an assumption cannot always be made. Sometimes, a specific dependence structure between sources can be assumed (or known), and merging rules corresponding to such structures can then be used $[8,20,15]$ (for example, an assumption of complete positive or negative correlation between the sources precision).

However, assuming that the (in)dependence structure between sources is well known is often unrealistic. In those cases, an alternative is to adopt a conservative approach when merging belief functions (i.e. by adding no extra information nor assumption in the combination process, or the least possible amount of it). Adopting such a cautious attitude is equivalent to applying the "least commitment principle", which informally states that one should never presuppose more beliefs than justified. This principle is basic in the frameworks of possibility theory, imprecise probability [24], and the Transferable Belief Model (TBM) [23]. It can be naturally exploited for the cautious merging of belief functions. 
This cautious approach can be interpreted and used in different ways. For instance, Denoeux [6] proposes a cautious conjunctive rule of combination based on Smets [21] canonical decomposition. Cattaneo [2] proposes to first consider the set of merged belief functions minimizing the resulting conflict (hence maximizing the result coherence) and then to select the most cautious belief function among them. Recently [7], we have proposed to maximize expected cardinality of belief functions in order to find a cautiously merged belief function. Although different, all these approaches agree on the fact that a cautious conjunctive merging rule should satisfy the property of idempotence, as this property ensures that the same information supplied by two dependent sources will remain unchanged after merging.

There are mainly three strategies to construct idempotent rules that make sense in the belief function setting. The first one looks for idempotent rules that satisfy a certain number of desired properties and appear sensible in the framework of belief functions. This is the solution retained by Denoeux [6] and Cattaneo [2]. The second strategy relies on the natural idempotent rule consisting of intersecting sets of probabilities and tries to express it in the particular case of belief functions (Chateauneuf [3]). Finally, the third approach, explored in this paper, starts from the natural idempotent rule in a less general framework, possibility theory, trying to extend it to belief functions. Namely, we study the generalisation of the minimum rule, viewing possibility distributions as contour functions of consonant belief functions [19]. If we denote $\left(m_{1}, \mathcal{F}_{1}\right),\left(m_{2}, \mathcal{F}_{2}\right)$ two belief functions, $\mathcal{P}_{1}, \mathcal{P}_{2}$ two sets of probabilities, and $\pi_{1}, \pi_{2}$ two possibility distributions, the three approaches are summarized in Figure 1 below.

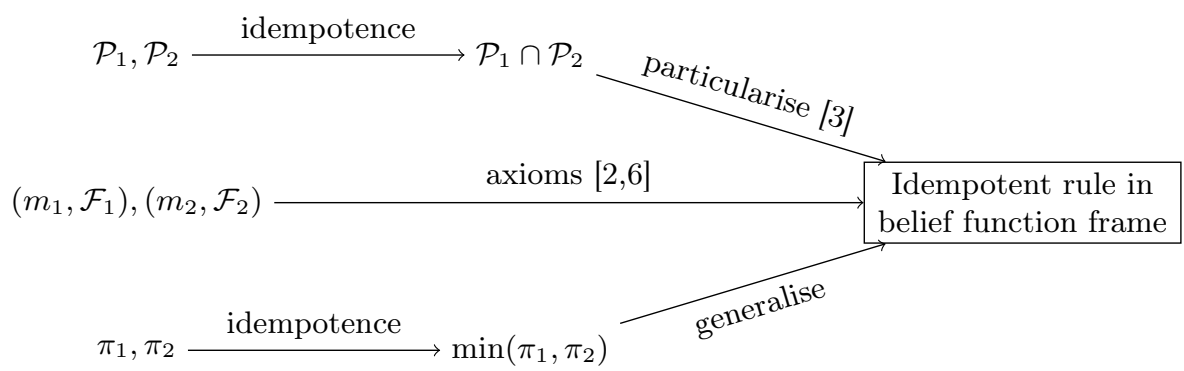

Fig. 1. Search of idempotent merging rules

Section 2 recalls basics of belief functions and defines conjunctive merging in this framework. Section 3 then studies to what extent the minimum rule of possibility theory can be extended to the framework of belief functions. The idea is to request that the contour function after merging be the minimum of the contour functions of the input belief functions, what we call the strong contour function principle. Note that a similar property holds for the unnormalized Dempster rule with respect to the product of contour functions. In the case of the minimum 
rule, we are lead to propose a weak contour principle as the former condition turns out to be too strong.

\section{Preliminaries}

This section introduces the basical tools concerning belief functions needed in this paper: their structure,the notions of inclusions and of conjunctive merging, as well as their link with other frameworks (possibility theory, convex sets of probabilities).

In the whole paper, we consider that information pertains to a variable $V$ taking its values on a finite space $\mathcal{V}$, with generic element denoted $v$.

\subsection{Belief functions}

Here, we assume that our belief state is modelled by a belief function, or, equivalently, by a basic belief assignment (bba).

Definition 1 (Basic belief assignment). A basic belief assignment (bba) is a function $m$ from the power set $2^{|\mathcal{V}|}$ of $\mathcal{V}$ to $[0,1]$ such that $\sum_{A \subseteq \mathcal{V}} m(A)=1$.

We denote by $\mathcal{M}_{\mathcal{V}}$ the set of bba's on $2^{|\mathcal{V}|}$. A set $A$ such that $m(A)>0$ is called a focal set. We denote by $\mathcal{F}$ the set of focal sets corresponding to bba $m$, and $(m, \mathcal{F})$ a complete belief structure. The number $m(A)>0$ is the mass of $A$. Given a bba $m$, belief, plausibility and commonality functions of an event $E \subseteq \mathcal{V}$ are, respectively

$$
\begin{aligned}
\operatorname{bel}(E) & =\sum_{A \subseteq E} m(A) \\
p l(E) & =\sum_{A \cap E \neq \emptyset} m(A)=1-\operatorname{bel}\left(E^{c}\right) \\
q(E) & =\sum_{E \subseteq A} m(A)
\end{aligned}
$$

A belief function measures to what extent an event is directly supported by the available information, while a plausibility function measures the maximal amount of evidence that could support a given event. A commonality function measures the quantity of mass that may be re-allocated to a particular set from its supersets. The commonality function increases when bigger focal sets receive greater mass assignments, hence the greater the commonality degrees, the less informative is the belief function. It can be shown [19] that any of the four representations, namely bbas, belief, plausibility and commonality functions contains the same amount of information. That is, from the (complete) knowledge of any of them, all the others can be retrieved by bijective transformations.

Note that in Shafer's seminal work [19], extensively taken over by Smets in his Transferable Belief Model [23], there are no references to any underlying 
probabilistic interpretation or framework. The bba and the associated belief function model beliefs per se. The mass given to $A$ measures the amount of belief exactly given to $A$, that is the amount of belief that $w \in A$, without being able to be more precise.

However, a belief structure $(m, \mathcal{F})$ can also be interpreted as a convex set of probabilities [24] $\mathcal{P}_{(m, \mathcal{F})}$ such that $\operatorname{Bel}(A)$ and $\operatorname{Pl}(A)$ are probability bounds:

$$
\mathcal{P}_{(m, \mathcal{F})}=\{P \mid \forall A \subset \mathcal{V}, \operatorname{Bel}(A) \leq P(A) \leq P l(A)\} .
$$

Classical probability distributions are retrieved when only singletons receive positive masses. This interpretation is closer to random sets and to Dempster's view [4]

\subsection{Possibility distributions and contour functions}

A possibility distribution $[26,10]$ is a mapping $\pi: \mathcal{V} \rightarrow[0,1]$ such that $\pi(v)=1$ for at least one element $v \in \mathcal{V}$. Two dual functions (respectively the possibility and necessity function) can be defined from $\pi: \Pi(A)=\sup _{v \in A} \pi(v)$ and $N(A)=$ $1-\Pi\left(A^{c}\right)$. Their characteristic properties are that, for any pair $A, B \subseteq \mathcal{V}$, we have:

$$
\Pi(A \cup B)=\max (\Pi(A), \Pi(B)) ; \quad N(A \cap B)=\min (N(A), N(B)) .
$$

A contour function of a belief structure $(m, \mathcal{F})$ is defined as follows

Definition 2. The contour function $\pi_{m}$ of a belief structure $(m, \mathcal{F})$ is a mapping $\pi_{(m, \mathcal{F})}: \mathcal{V} \rightarrow[0,1]$ such that, for any $x \in \mathcal{V}$,

$$
\pi_{(m, \mathcal{F})}(v)=p l(\{v\})=q(\{v\})^{1},
$$

with $p l, q$ the plausibility and commonality functions of $(m, \mathcal{F})$.

A belief structure $(m, \mathcal{F})$ is called consonant when its focal sets are completely ordered with respect to inclusion (that is, for any $A, B \in \mathcal{F}$, we have either $A \subset B$ or $B \subset A$ ). In this case, the plausibility and belief functions have the characteristic properties of respectively possibility and necessity functions, and the information contained in the consonant belief structure can be represented by the possibility distribution whose mapping correspond to the contour function

$$
\pi(v)=\sum_{v \in E} m(E)
$$

Conversely, any possibility distribution and its associated possibility (resp. necessity) function defines a unique consonant belief structure and plausibility (resp. belief) function. If $\pi$ is a possibility distribution, and if $0=\alpha_{0} \leq \alpha_{1} \leq \ldots \leq$ $\ldots \alpha_{M}=1$ is the (finite) set of distinct values assumed by $\pi$ over $\mathcal{V}$, then the

\footnotetext{
${ }^{1}$ Note that the equality between $p l$ and $q$ on singletons always holds.
} 
corresponding belief structure $\left(m_{\pi}, \mathcal{F}_{\pi}\right)$ has, for $i=1, \ldots, M$, the following $M$ focal sets:

$$
\left\{\begin{array}{c}
E_{i}=\left\{v \in X \mid \pi(v) \geq \alpha_{i}\right\} \\
m\left(E_{i}\right)=\alpha_{i}-\alpha_{i-1},
\end{array}\right.
$$

with $m\left(E_{i}\right)$ the mass given to $E_{i}$.

For any belief structure $(m, \mathcal{F})$, the contour function can be seen as a (possibly unnormalized) possibility distribution, and is a trace of the whole belief structure $(m, \mathcal{F})$ restricted to singletons. Except when $(m, \mathcal{F})$ is consonant, the contour function represents only part of the information contained in $(m, \mathcal{F})$. The contour function is therefore a summary, easier to manipulate than the whole random set.

\subsection{Inclusion and information orderings between belief functions}

Inclusion relationships are natural tools to compare the informative contents of set-valued uncertainty representations. Let $A, B \subseteq \mathcal{V}$ be two sets, $\left(m_{A}, \mathcal{F}_{A}\right),\left(m_{B}, \mathcal{F}_{B}\right)$ the corresponding belief structures and $\pi_{A}, \pi_{B}$ the corresponding possibility distributions. Recall that a set $A$ can be represented by the belief structure such that $m(A)=1$ and the possibility distribution $\pi(v)=1$ if $v \in A$, zero otherwise. In this crisp case, all the following expressions are equivalent:

$-A \subseteq B$

$-\forall E \subseteq \mathcal{V}, p l_{A}(E) \leq p l_{B}(E)$,

$-\forall E \subseteq \mathcal{V}, q_{A}(E) \leq q_{B}(E)$,

$-\forall E \subseteq \mathcal{V}, \pi_{A}(v) \leq \pi_{B}(v)$.

When working with belief functions and bbas, all these equivalent notions have different counterparts [9], leading to the definitions of $x$-inclusions, with $x \in$ $\{p l, q, s, \pi\}$.

Definition 3 (pl-inclusion). A belief structure $\left(m_{1}, \mathcal{F}_{1}\right)$ defined on $\mathcal{V}$ is said to be pl-included in another belief structure $\left(m_{2}, \mathcal{F}_{2}\right)$ defined on $\mathcal{V}$ if and only if, for all $A \subseteq \mathcal{V}$,

$$
p l_{1}(A) \leq p l_{2}(A)
$$

and this relation is denoted by $\left(m_{1}, \mathcal{F}_{1}\right) \sqsubseteq_{p l}\left(m_{2}, \mathcal{F}_{2}\right)$ and by $\left(m_{1}, \mathcal{F}_{1}\right) \sqsubset_{p l}$ $\left(m_{2}, \mathcal{F}_{2}\right)$ if the above inequality is strict for at least one event.

We also have that $\left(m_{1}, \mathcal{F}_{1}\right)$ is pl-included in $\left(m_{2}, \mathcal{F}_{2}\right)$ if and only if $\mathcal{P}_{\left(m_{1}, \mathcal{F}_{1}\right)} \subseteq$ $\mathcal{P}_{\left(m_{2}, \mathcal{F}_{2}\right)}$, thus the notion of $p l$-inclusion is coherent with the interpretation of belief functions as probability bounds. Note that, due to the duality relation between plausibility and belief functions, $p l$-inclusion also covers the case of bel-inclusion.

Definition 4 (q-inclusion). A belief structure $\left(m_{1}, \mathcal{F}_{1}\right)$ defined on $\mathcal{V}$ is said to be q-included in another belief structure $\left(m_{2}, \mathcal{F}_{2}\right)$ defined on $\mathcal{V}$ if and only if, for all $A \subseteq \mathcal{V}$,

$$
q_{1}(A) \leq q_{2}(A)
$$


and this relation is denoted by $\left(m_{1}, \mathcal{F}_{1}\right) \sqsubseteq_{q}\left(m_{2}, \mathcal{F}_{2}\right)$ and by $\left(m_{1}, \mathcal{F}_{1}\right) \sqsubset_{q}\left(m_{2}, \mathcal{F}_{2}\right)$ if the above inequality is strict for at least one event.

As the commonality function is higher when higher masses are given to bigger sets, the notion of $q$-inclusion also allows to compare informative contents, but neither does imply nor is implied by the $p l$-inclusion [9]. Also, existing results tend to show that the notion of $q$-inclusion is more natural when working within the TBM interpretation $[14,7]$.

Definition 5 (s-inclusion). A belief structure $\left(m_{1}, \mathcal{F}_{1}\right)$ defined on $\mathcal{V}$ with $\mathcal{F}_{1}=$ $\left\{E_{1}, \ldots, E_{q}\right\}$ is said to be s-included in another belief structure $\left(m_{2}, \mathcal{F}_{2}\right)$ defined on $\mathcal{V}$ with $\mathcal{F}_{2}=\left\{E_{1}^{\prime}, \ldots, E_{p}^{\prime}\right\}$ if and only if there exists a non-negative matrix $G$ of generic term $g_{i j}$ such that

$$
\begin{gathered}
\text { for } j=1, \ldots, p, \quad \sum_{i=1}^{q} g_{i j}=1, \\
g_{i j}>0 \Rightarrow E_{i} \subseteq E_{j}^{\prime}, \\
\sum_{j=1}^{p} m_{2}\left(E_{j}^{\prime}\right) g_{i j}=m_{1}\left(E_{i}\right) .
\end{gathered}
$$

This relation is denoted by $\left(m_{1}, \mathcal{F}_{1}\right) \sqsubseteq_{s}\left(m_{2}, \mathcal{F}_{2}\right)$ and by $\left(m_{1}, \mathcal{F}_{1}\right) \sqsubset_{s}\left(m_{2}, \mathcal{F}_{2}\right)$ if there is at least a pair $i, j$ such that $g_{i j}>0$ and $E_{i} \subset E_{j}$.

The term $g_{i j}$ is the proportion of the focal set $E_{j}^{\prime}$ that "flows down" to focal set $E_{i}$. In other words, $\left(m_{1}, \mathcal{F}_{1}\right)$ is s-included in $\left(m_{2}, \mathcal{F}_{2}\right)$ if the mass of any focal set $E_{j}^{\prime}$ of $\left(m_{2}, \mathcal{F}_{2}\right)$ can be redistributed among subsets of $E_{j}^{\prime}$ in $\left(m_{1}, \mathcal{F}_{1}\right)$.

Definition 6 ( $\pi$-inclusion). A belief structure $\left(m_{1}, \mathcal{F}_{1}\right)$ defined on $\mathcal{V}$ is said to be $\pi$-included in another belief structure $\left(m_{2}, \mathcal{F}_{2}\right)$ defined on $\mathcal{V}$ if and only if, for all $x \in \mathcal{V}$,

$$
\pi_{\left(m_{1}, \mathcal{F}_{1}\right)}(x) \leq \pi_{\left(m_{2}, \mathcal{F}_{2}\right)}(x)
$$

and this relation is denoted $\left(m_{1}, \mathcal{F}_{1}\right) \sqsubseteq \pi\left(m_{2}, \mathcal{F}_{2}\right)$ and by $\left(m_{1}, \mathcal{F}_{1}\right) \sqsubset_{\pi}\left(m_{2}, \mathcal{F}_{2}\right)$ if the above inequality is strict for at least one element.

The notion of $\pi$-inclusion is the extension to general belief structures of the notion of specificity between possibility distributions (a possibility distribution $\pi_{1}$ is more specific, or included in another possibility distribution $\pi_{2}$ if $\pi_{1} \leq \pi_{2}$ ).

Since notions of inclusion allow to compare informative contents, we will also say, when $\left(m_{1}, \mathcal{F}_{1}\right) \sqsubseteq_{x}\left(m_{2}, \mathcal{F}_{2}\right)\left(\left(m_{1}, \mathcal{F}_{1}\right) \sqsubset_{x}\left(m_{2}, \mathcal{F}_{2}\right)\right)$ with $x \in\{p l, q, s, \pi\}$, that $\left(m_{1}, \mathcal{F}_{1}\right)$ is (strictly) $x$-more committed than $\left(m_{2}, \mathcal{F}_{2}\right)$. The following implications hold between these notions of inclusion [9]:

$$
\left(m_{1}, \mathcal{F}_{1}\right) \sqsubseteq_{s}\left(m_{2}, \mathcal{F}_{2}\right) \Rightarrow\left\{\begin{array}{c}
\left(m_{1}, \mathcal{F}_{1}\right) \sqsubseteq_{p l}\left(m_{2}, \mathcal{F}_{2}\right) \\
\left(m_{1}, \mathcal{F}_{1}\right) \sqsubseteq_{q}\left(m_{2}, \mathcal{F}_{2}\right)
\end{array}\right\} \Rightarrow\left(m_{1}, \mathcal{F}_{1}\right) \sqsubseteq_{\pi}\left(m_{2}, \mathcal{F}_{2}\right) .
$$


As classical inclusion does with crisp sets, each of these notions induces a partial ordering between elements of $\mathcal{M}_{\mathcal{V}}$. Note that the relation $\sqsubseteq \pi$ only induces a partial pre-order (i.e., we can have $\left(m_{1}, \mathcal{F}_{1}\right) \sqsubseteq_{\pi}\left(m_{2}, \mathcal{F}_{2}\right)$ and $\left(m_{2}, \mathcal{F}_{2}\right) \sqsubseteq_{\pi}\left(m_{1}, \mathcal{F}_{1}\right)$ with $\left.\left(m_{1}, \mathcal{F}_{1}\right) \neq\left(m_{1}, \mathcal{F}_{1}\right)\right)$, while the others induce partial orders (i.e., they are antisymmetric). This is due to the fact that the notion of $\pi$-inclusion is based on the contour function that does not, in general, contain all the information contained in a belief structure. Note that, since notions of $p l, q$ and $s$-inclusion are antisymmetric, we also have

$$
\left(m_{1}, \mathcal{F}_{1}\right) \sqsubset_{s}\left(m_{2}, \mathcal{F}_{2}\right) \Rightarrow\left\{\begin{array}{c}
\left(m_{1}, \mathcal{F}_{1}\right) \sqsubset_{p l}\left(m_{2}, \mathcal{F}_{2}\right) \\
\left(m_{1}, \mathcal{F}_{1}\right) \sqsubset_{q}\left(m_{2}, \mathcal{F}_{2}\right)
\end{array}\right.
$$

The following example illustrates the fact that $\pi$-inclusion not being antisymmetric, we can have strict $q$-inclusion and $p l$-inclusion while having equality for these two functions on singletons.

Example 1. Consider the two following possibility distributions $\pi_{1}, \pi_{2}$, expressed as belief structures $\left(m_{1}, \mathcal{F}_{1}\right),\left(m_{2}, \mathcal{F}_{2}\right)$ on the domain $\mathcal{V}=\left\{x_{1}, x_{2}, x_{3}\right\}$

\begin{tabular}{cccc}
$\left(m_{1}, \mathcal{F}_{1}\right)$ & & $\left(m_{2}, \mathcal{F}_{2}\right)$ & \\
Focal sets & Mass & Focal sets & Mass \\
\hline$E_{11}=\left\{x_{2}\right\}$ & 0.5 & $E_{21}=\left\{x_{2}, x_{3}\right\}$ & 0.5 \\
$E_{12}=\left\{x_{1}, x_{2}, x_{3}\right\}$ & 0.5 & $E_{22}=\left\{x_{1}, x_{2}\right\}$ & 0.5
\end{tabular}

These two random sets have the same contour function, while $\left(m_{1}, \mathcal{F}_{1}\right) \sqsubset_{p l}$ $\left(m_{2}, \mathcal{F}_{2}\right)$ and $\left(m_{2}, \mathcal{F}_{2}\right) \sqsubset_{q}\left(m_{1}, \mathcal{F}_{1}\right)$.

The above example can be generalized, in the sense that if we have two belief functions $m_{1}, m_{2}$ such that $m_{1} \sqsubset_{q} m_{2}$ and $m_{1} \sqsubset_{q} m_{2}$, we have the following implications:

$$
\begin{aligned}
& m_{1} \sqsubset_{q} m_{2} \Rightarrow m_{1} \sqsubseteq_{\pi} m_{2}, \\
& m_{2} \sqsubset_{p l} m_{1} \Rightarrow m_{2} \sqsubseteq_{\pi} m_{1},
\end{aligned}
$$

and this implies that $m_{1}={ }_{\pi} m_{2}$.

If belief structures are consonant, then all the notions of $p l, q, s$ and $\pi$ inclusion reduce to the same definition (that is, the one of $\pi$-inclusion).

Recently, Denoeux [6] has introduced other notions of inclusion, namely the $w$-inclusion and $v$-inclusion, based on Smets canonical decomposition [21]. They also induce partial orders between belief functions. Given a belief structure $(m, \mathcal{F})$ for which $m(\mathcal{V}) \neq 0$, the $w$-transformation affects to each subset $A \subseteq \mathcal{V}$ the value $w(A) \in(0,+\infty)$ such that

$$
w(A)=\prod_{B \supseteq A} q(B)^{|B|-|A|+1} .
$$

A belief structure $\left(m_{1}, \mathcal{F}_{1}\right)$ is said to be w-included in another belief structure $\left(m_{2}, \mathcal{F}_{2}\right)$ if $w_{1}(A) \leq w_{2}(A)$ for any $A \subseteq \mathcal{V}$. When $(m, \mathcal{F})$ is consonant and has $\pi$ for contour function, if we note $\pi_{k}=\pi\left(v_{k}\right)$ with a ranking of elements 
$\mathcal{V}=\left\{v_{1}, \ldots, v_{K}\right\}$ such that $1=\pi_{1} \geq \pi_{2} \geq \ldots \geq \pi_{K}$, and $A_{k}=\left\{v_{1}, \ldots, v_{k}\right\}$, its $\mathrm{w}$-transformation is

$$
w(A)= \begin{cases}\frac{\pi_{k+1}}{\pi_{k}}, & A=A_{k}, 1 \leq k<K \\ 1, & \text { otherwise. }\end{cases}
$$

We will not consider these orderings here, for the reason that the notion of $w$ inclusion does not reduce to the notion of $\pi$-inclusion when considering only consonant random sets, as shows the next example.

Example 2. Consider the two possibility distributions $\pi_{1}, \pi_{2}$ on space $\mathcal{V}=\left\{v_{1}, v_{2}, v_{3}, v_{4}\right\}$ summarized in the following table

\begin{tabular}{|c|c|}
\hline$\pi_{1}$ & $\pi_{2}$ \\
\hline $\begin{array}{llll}v_{1} & v_{2} & v_{3} & v_{4}\end{array}$ & $\begin{array}{llll}v_{1} & v_{2} & v_{3} & v_{4}\end{array}$ \\
\hline 0.10 .50 .61 & 0.150 .811 \\
\hline
\end{tabular}

we do have $\pi_{1} \sqsubset \pi_{2}$ but $w_{1}\left(\left\{v_{1}, v_{2}\right\}\right)=5 / 6>w_{2}\left(\left\{v_{1}, v_{2}\right\}\right)=8 / 10$ and $w_{1}\left(\left\{v_{1}, v_{2}, v_{3}\right\}\right)=$ $6 / 10<w_{2}\left(\left\{v_{1}, v_{2}, v_{3}\right\}\right)=1$, hence the corresponding random set are $w$-incomparable.

As all these notions induce partial orders between belief structures, it is sometimes desirable (for example, when one has to select a single least-specific belief structure among a set of such structures) to use alternative criteria inducing complete ordering between belief structures. One of such criteria, that is used in other approaches to the cautious merging of belief function [14,7], is the expected cardinality of a belief structure $(m, \mathcal{F})$, that we denote by $\mathbb{C}_{|(m, \mathcal{F})|}$ and whose value is

$$
\mathbb{C}_{|(m, \mathcal{F})|}=\sum_{E \in \mathcal{F}} m(E)|E|,
$$

which is also equal to the cardinality of the contour function $\pi_{(m, \mathcal{F})}$ [12], that is

$$
\mathbb{C}_{|(m, \mathcal{F})|}=\sum_{x \in \mathcal{V}} \pi_{(m, \mathcal{F})}(x)
$$

Yet other information comparison measures exist [2,17], but since our aim is to generalize merging rules coming from possibility theory, expected cardinality appears to be the best choice, due to the Equality (4). We can thus define the notion of cardinality specificity:

Definition 7 (C-specificity). A belief structure $\left(m_{1}, \mathcal{F}_{1}\right)$ defined on $\mathcal{V}$ is said to be more $\mathbb{C}$-specific than another belief structure $\left(m_{2}, \mathcal{F}_{2}\right)$ defined on $\mathcal{V}$ if and only if we have the inequality

$$
\mathbb{C}_{\left|\left(m_{1}, \mathcal{F}_{1}\right)\right|} \leq \mathbb{C}_{\left|\left(m_{2}, \mathcal{F}_{2}\right)\right|}
$$

and this relation is denoted $\left(m_{1}, \mathcal{F}_{1}\right) \sqsubseteq \mathbb{C}\left(m_{2}, \mathcal{F}_{2}\right)$ and by $\left(m_{1}, \mathcal{F}_{1}\right) \sqsubset \mathbb{C}\left(m_{2}, \mathcal{F}_{2}\right)$ if the above inequality is strict. 
The following lemma also indicates that the order between belief structures induced by $\mathbb{C}$-specificity is in agreement with the other inclusion notions used in this paper.

Lemma 1. Let $\left(m_{1}, \mathcal{F}_{1}\right),\left(m_{2}, \mathcal{F}_{2}\right)$ be two random sets. Then, the following implications holds:

$$
\begin{gathered}
I\left(m_{1}, \mathcal{F}_{1}\right) \sqsubset_{\pi}\left(m_{2}, \mathcal{F}_{2}\right) \rightarrow\left(m_{1}, \mathcal{F}_{1}\right) \sqsubset_{\mathbb{C}}\left(m_{2}, \mathcal{F}_{2}\right) \\
I I\left(m_{1}, \mathcal{F}_{1}\right) \sqsubset_{s}\left(m_{2}, \mathcal{F}_{2}\right) \rightarrow\left(m_{1}, \mathcal{F}_{1}\right) \sqsubset_{\mathbb{C}}\left(m_{2}, \mathcal{F}_{2}\right) \\
I I I\left(m_{1}, \mathcal{F}_{1}\right) \sqsubset_{p l}\left(m_{2}, \mathcal{F}_{2}\right) \rightarrow\left(m_{1}, \mathcal{F}_{1}\right) \sqsubseteq_{\mathbb{C}}\left(m_{2}, \mathcal{F}_{2}\right) \\
I V\left(m_{1}, \mathcal{F}_{1}\right) \sqsubset_{q}\left(m_{2}, \mathcal{F}_{2}\right) \rightarrow\left(m_{1}, \mathcal{F}_{1}\right) \sqsubseteq_{\mathbb{C}}\left(m_{2}, \mathcal{F}_{2}\right)
\end{gathered}
$$

Proof. I Immediate, since $\pi_{\left(m_{1}, \mathcal{F}_{1}\right)}<\pi_{\left(m_{2}, \mathcal{F}_{2}\right)}$ implies the same strict inequality between $\mathbb{C}_{\left|\left(m_{1}, \mathcal{F}_{1}\right)\right|}$ and $\mathbb{C}_{\left|\left(m_{2}, \mathcal{F}_{2}\right)\right|}$ (see Eq. (4))

II Since $s$-inclusion implies $\pi$-inclusion, we have $\left(m_{1}, \mathcal{F}_{1}\right) \sqsubset_{s}\left(m_{2}, \mathcal{F}_{2}\right) \rightarrow$ $\left(m_{1}, \mathcal{F}_{1}\right) \sqsubseteq \mathbb{C}\left(m_{2}, \mathcal{F}_{2}\right)$. To see that the second inequality is strict, simply consider the fact that, if $\left(m_{1}, \mathcal{F}_{1}\right) \sqsubset_{s}\left(m_{2}, \mathcal{F}_{2}\right)$ and if we consider $\mathcal{F}_{1}=\left\{E_{1}, \ldots, E_{q}\right\}$ and $\mathcal{F}_{2}=\left\{E_{1}^{\prime}, \ldots, E_{p}^{\prime}\right\}$, then

$$
\mathbb{C}_{\left|\left(m_{1}, \mathcal{F}_{1}\right)\right|}=\sum_{i=1}^{q} m_{1}\left(E_{i}\right)\left|E_{i}\right|=\sum_{i=1}^{q} \sum_{j=1}^{p} m_{2}\left(E_{j}^{\prime}\right) g_{i j}\left|E_{i}\right| .
$$

Since $E_{i} \subseteq E_{j}^{\prime}$ if $g_{i j}>0$, and at least one $g_{i j}>0$ such that $E_{i} \subset E_{j}^{\prime}$, the sum (5) is strictly lower than $\mathbb{C}_{\left|\left(m_{2}, \mathcal{F}_{2}\right)\right|}=\sum_{j=1}^{p} m_{2}\left(E_{j}^{\prime}\right)\left|E_{j}^{\prime}\right|$. This is sufficient to show that $\left(m_{1}, \mathcal{F}_{1}\right) \sqsubset \mathbb{C}\left(m_{2}, \mathcal{F}_{2}\right)$ (due to Eq (4)).

III The implication is immediate, given Eq (4) and the fact that $p l_{1} \leq p l_{2}$. Example 3 indicates that two strictly $p l$-included belief structures can have equal cardinality.

IV Immediate, with the same arguments applied to $q$-inclusion.

\subsection{Conjunctive merging and least commitment}

Let $\left(m_{1}, \mathcal{F}_{1}\right),\left(m_{2}, \mathcal{F}_{2}\right)$ be two belief structures defined on $\mathcal{V}$, supplied by two, not necessarily independent, sources (e.g., two experts potentially sharing some common opinions, two physical models based on similar equations). We define a belief structure $\left(m_{\cap}, \mathcal{F}_{\cap}\right)$ resulting from a conjunctive merging of $\left(m_{1}, \mathcal{F}_{1}\right),\left(m_{2}, \mathcal{F}_{2}\right)$ as the result of the following procedure [7]:

1. A joint mass distribution $m$ is built on $\mathcal{V}^{2}$, having focal sets of the form $A \times B$ where $A \in \mathcal{F}_{1}, B \in \mathcal{F}_{2}$ and preserving $m_{1}, m_{2}$ as marginals. It means that

$$
\begin{aligned}
& \forall A \in \mathcal{F}_{1}, m_{1}(A)=\sum_{B \in \mathcal{F}_{2}} m(A, B), \\
& \forall B \in \mathcal{F}_{2}, m_{2}(B)=\sum_{A \in \mathcal{F}_{1}} m(A, B) .
\end{aligned}
$$


2. Each joint mass $m(A, B)$ is allocated to the subset $A \cap B$ only.

We call a merging rule satisfying these two conditions conjunctive ${ }^{2}$, and denote $\mathcal{M}_{12}$ the set of conjunctively merged belief structures. The idea behind the conjunctive approach is to gain as much information as possible from the fusion process. Not every belief structure $\left(m_{\cap}, \mathcal{F}_{\cap}\right)$ obtained by conjunctive merging is normalized (i.e. one may get $m(\emptyset) \neq 0$ ). In this paper, unless stated otherwise, we do not assume that a conjunctively merged belief structure has to be normalised. We also do not renormalise such belief structures, because, after renormalisation, they no longer satisfy Eq. (6), and renormalisation is usually not required when working with possibility distributions. A belief structure $\left(m_{\cap}, \mathcal{F}_{\cap}\right)$ on $\mathcal{V}$ obtained by a conjunctive merging rule is a specialisation of both $\left(m_{1}, \mathcal{F}_{1}\right)$ and $\left(m_{2}, \mathcal{F}_{2}\right)$ , and $\mathcal{M}_{12}$ is a subset of all belief structures that are specialisations of both $\left(m_{1}, \mathcal{F}_{1}\right)$ and $\left(m_{2}, \mathcal{F}_{2}\right)$, that is

$$
\mathcal{M}_{12} \subseteq\left\{m \in \mathcal{M}_{\mathcal{V}} \mid i=1,2, \quad m \sqsubseteq_{s} m_{i}\right\},
$$

with the inclusion being usually strict ${ }^{3}$. Regarding the belief structures inside $\mathcal{M}_{12}$, three situations can occur:

1. $\mathcal{M}_{12}$ contains only normalized belief functions. It means that $\forall A \in \mathcal{F}_{1}, B \in$ $\mathcal{F}_{2}, A \cap B \neq \emptyset$. Only in that case does the result of merging by Dempster rule of combination is normalized. The two bbas are said to be logically consistent.

2. $\mathcal{M}_{12}$ contains both subnormalized and normalized bbas. It means that $\exists A, B, A \cap$

$B=\emptyset$ and that the marginal-preservation Equations (6) have solutions which allocate zero mass $m(A, B)$ to such $A \times B$. The two bbas are said to be nonconflicting. Chateauneuf [3] have shown that being non-conflicting is a sufficient and necessary condition for $\mathcal{P}_{\left(m_{1}, \mathcal{F}_{1}\right)} \cap \mathcal{P}_{\left(m_{2}, \mathcal{F}_{2}\right)}$ to be non-empty, and that for each subset $A \subseteq \mathcal{V}$, the lower probability $\underline{P}(A)$ of $\mathcal{P}_{\left(m_{1}, \mathcal{F}_{1}\right)} \cap \mathcal{P}_{\left(m_{2}, \mathcal{F}_{2}\right)}$ is equal to the lowest belief measure on $A$ induced by all normalized belief structures in $\mathcal{M}_{12}$.

3. $\mathcal{M}_{12}$ contains only subnormalized belief functions. This situation is equivalent to having $\mathcal{P}_{\left(m_{1}, \mathcal{F}_{1}\right)} \cap \mathcal{P}_{\left(m_{2}, \mathcal{F}_{2}\right)}=\emptyset$. The two bbas are said to be conflicting.

Note that, in the first situation, the lower probability of $\mathcal{P}_{\left(m_{1}, \mathcal{F}_{1}\right)} \cap \mathcal{P}_{\left(m_{2}, \mathcal{F}_{2}\right)}$ is equal to the lowest belief measures induced by the set of all belief structures in $\mathcal{M}_{12}$ (since they are all normalised).

When both sources can be considered as independent, the TBM conjunctive rule consists of taking the merged belief structure inside $\mathcal{M}_{12}$ that take the product of masses (i.e., $m(A, B)=m_{1}(A) \cdot m_{2}(B)$ in Equations $\left.(6)\right)$ as the joint mass.

\footnotetext{
${ }^{2}$ A disjunctive merging rule can be defined likewise, changing $\cap$ into $\cup$.

${ }^{3}$ Consider, for example, the empty belief structures $m_{1}(\mathcal{V})=m_{2}(\mathcal{V})=1$ as marginals. The set of specialisation of both of them is every possible belief structure, while only the empty one can be reached by the conjunctive merging defined in this paper
} 
However, when sources cannot be considered as independent and when the dependence structure between them is not well known, a common practice is to use the principle of least-commitment to build the merged belief structure. That is, to adopt a cautious attitude. Let us note $\mathcal{M}_{12}^{\stackrel{5}{x}}$ the set of all maximal elements inside $\mathcal{M}_{12}$ when they are ordered with respect to $x$-inclusion, with $x \in\{s, p l, q, \pi, \mathbb{C}\}$. Least-commitment principle then often consists of choosing a given $x$ and picking a particular element inside $\mathcal{M} \underset{12}{\sqsubseteq \mathrm{x}}$ that also satisfies a number of desired properties.

Since idempotence is often considered as a natural property to satisfy when cautiously merging multiple sources of information, another option is to search how to adapt idempotent rules coming from other frameworks to the merging of belief structures. Chateauneuf [3] has already explored the links between the natural idempotent rule used to merge sets of probabilities and belief structures. In the rest of the paper, we will study how to extend the natural idempotent and cautious minimum rule originating from possibility theory, and under which conditions the conjunctive merging of belief structures can satisfy such an extension.

\section{$3 \quad$ Extending possibilistic idempotent rule}

If $\pi_{1}, \pi_{2}$ denote two possibility distributions, the natural conjunctive idempotent rule between these two distributions is the minimum $\min \left(\pi_{1}, \pi_{2}\right)$. It can also be seen as the most cautious, as the minimum is the most conservative of all tnorms [16].

Now, let us consider two random sets $\left(m_{1}, \mathcal{F}_{1}\right),\left(m_{2}, \mathcal{F}_{2}\right)$ and their respective contour functions $\pi_{\left(m_{1}, \mathcal{F}_{1}\right)}, \pi_{\left(m_{2}, \mathcal{F}_{2}\right)}$. First notice the following property:

Proposition 1 (s-covering). Let $\left(m_{1}, \mathcal{F}_{1}\right),\left(m_{2}, \mathcal{F}_{2}\right)$ be two belief structures. Then, the following inequality holds for any $v \in \mathcal{V}$ :

$$
\max _{(m, \mathcal{F}) \in \mathcal{M}_{12}} \pi_{(m, \mathcal{F})}(v) \leq \min \left(\pi_{\left(m_{1}, \mathcal{F}_{1}\right)}(v), \pi_{\left(m_{2}, \mathcal{F}_{2}\right)}(v)\right)
$$

Proof. Since any element $(m, \mathcal{F})$ in $\mathcal{M}_{12}$ are s-included in $\left(m_{1}, \mathcal{F}_{1}\right)$ and $\left(m_{2}, \mathcal{F}_{2}\right)$, and $s$-inclusion implies $\pi$-inclusion (Equation (2)), we have for any $x \in \mathcal{V}$ and any $(m, \mathcal{F}) \in \mathcal{M}_{12}$ that $\pi_{(m, \mathcal{F})}(v) \leq \min \left(\pi_{\left(m_{1}, \mathcal{F}_{1}\right)}(v), \pi_{\left(m_{2}, \mathcal{F}_{2}\right)}(v)\right)$ with $\pi_{(m, \mathcal{F})}, \pi_{\left(m_{1}, \mathcal{F}_{1}\right)}, \pi_{\left(m_{2}, \mathcal{F}_{2}\right)}$ the contour functions of $(m, \mathcal{F}),\left(m_{1}, \mathcal{F}_{1}\right),\left(m_{2}, \mathcal{F}_{2}\right)$. Since this is true for all elements of $\mathcal{M}_{12}$, this is enough to prove (7).

It is known [18] that the same inequality holds for sets of probabilities, since, given two such sets $\mathcal{P}_{1}, \mathcal{P}_{2}$, their respective upper probabilities $\bar{P}_{1}, \bar{P}_{2}$, their intersection $\mathcal{P}_{1} \cap \mathcal{P}_{2}$ and the induced lower probability $\bar{P}_{12}$, we have, for all events $A \subseteq \mathcal{V}, \bar{P}_{12}(A) \leq \min \left(\bar{P}_{1}(A), \bar{P}_{2}(A)\right)^{4}$. Considering the idempotent rule

\footnotetext{
${ }^{4}$ Note that the present situation is a bit different, since merging of probability sets do not allow for unnormalized belief structures, while we do allow for such belief structures here.
} 
of possibility theory and this property, it makes sense to ask for inequality (7) to become an equality. We now study two different ways to formulate this requirement on conjunctively merged belief structures.

\subsection{Strong contour function principle (SCFP)}

Let us first start with the strongest requirement.

Definition 8 (Strong contour function principle (SCFP)). Let $\left(m_{1}, \mathcal{F}_{1}\right),\left(m_{2}, \mathcal{F}_{2}\right)$ be two belief structures and $\mathcal{M}_{12}$ the set of conjunctively merged belief structures. Then, an element $\left(m_{1 \wedge 2}, \mathcal{F}_{1 \wedge 2}\right)$ in $\mathcal{M}_{12}$ is said to satisfy the strong contour function principle if, for any $v \in \mathcal{V}$,

$$
\pi_{\left(m_{1 \wedge 2}, \mathcal{F}_{1 \wedge 2}\right)}(v)=\min \left(\pi_{\left(m_{1}, \mathcal{F}_{1}\right)}(x), \pi_{\left(m_{2}, \mathcal{F}_{2}\right)}(v)\right),
$$

with $\pi_{\left(m_{1 \wedge 2}, \mathcal{F}_{1 \wedge 2}\right)}(v)$ the contour function of $\left(m_{1 \wedge 2}, \mathcal{F}_{1 \wedge 2}\right)(v)$.

That is, we require that the selected merged belief structure should have a contour functions equal to the minimum of the two marginal contour functions. It is clearly an extension of the minimum rule of possibility theory, since we retrieve it if both $\left(m_{1}, \mathcal{F}_{1}\right),\left(m_{2}, \mathcal{F}_{2}\right)$ are consonant.

Also observe that, given Eq (4), a merged belief structure satisfying the SCFP would also have a maximal expected cardinality among elements of $\mathcal{M}_{12}$, and hence be coherent with previous approaches [7] studying the maximisation of expected cardinality as a choice criteria for the merged belief structure.

Let us first assume that the two belief structures $\left(m_{1}, \mathcal{F}_{1}\right),\left(m_{2}, \mathcal{F}_{2}\right)$ are such that the SCFP can be satisfied. In this case, satisfying SCFP also imply satisfying the two following properties.

Proposition 2 (idempotence). Let $\left(m_{1}, \mathcal{F}_{1}\right)=\left(m_{2}, \mathcal{F}_{2}\right)=(m, \mathcal{F})$ be two identical belief structures. Then, the unique element in $\mathcal{M}_{12}$ satisfying Equation (8) is

$$
\left(m_{1 \wedge 2}, \mathcal{F}_{1 \wedge 2}\right)=(m, \mathcal{F})
$$

Proof. That $(m, \mathcal{F})$ is a solution of Equation (8) is immediate, since for any $v \in \mathcal{V}$ we have $p l_{1}(\{v\})=p l_{2}(\{v\})=p l(\{v\})$.

We must now show that any other belief structure in $\mathcal{M}_{12}$ is not a solution of (8). Consider a belief structure $\left(m_{\prime}, \mathcal{F}_{1}\right)$ that is a specialization of $(m, \mathcal{F})$ and is not $(m, \mathcal{F})$. Let $\mathcal{F}_{1}=\left\{E_{1}, \ldots, E_{p}\right\}$ be the focal sets of $(m, \mathcal{F})$. If $\left(m, \mathcal{F}_{1}\right)$ is not equal to $(m, \mathcal{F})$, this implies that there are at least two distinct focal sets $E_{i}, E_{j}$ such that a non-null weight $q_{i j}$ is given to $E_{i} \cap E_{j}$. Since $E_{i}$ and $E_{j}$ are distinct, there is at least an element $x \in X$ such that $x \notin E_{i} \cap E_{j}$, while $x \in E_{i}$ or $x \in E_{j}$, and for that element we have $p l_{1}(x) \geq p l^{\prime}(x)-q_{i j}$. Consequently, Equation $(8)$ can only be satisfied if we take $\left(m_{1 \wedge 2}, \mathcal{F}_{1 \wedge 2}\right)=(m, \mathcal{F})$.

This indicates that the SCFP is a sufficient condition to ensure that a merging rule is idempotent. 
Proposition 3 (s-coherence). Let $\left(m_{1}, \mathcal{F}_{1}\right)$ be (strictly) s-included in $\left(m_{2}, \mathcal{F}_{2}\right)$, that is $\left(m_{1}, \mathcal{F}_{1}\right) \sqsubset_{s}\left(m_{2}, \mathcal{F}_{2}\right)$. Then, the unique element in $\mathcal{M}_{12}$ satisfying Equation (8) is

$$
\left(m_{1 \wedge 2}, \mathcal{F}_{1 \wedge 2}\right)=\left(m_{1}, \mathcal{F}_{1}\right) .
$$

Proof. First, if $\left(m_{1}, \mathcal{F}_{1}\right) \sqsubset_{s}\left(m_{2}, \mathcal{F}_{2}\right)$, this means that $p l_{1}(\{v\}) \leq p l_{2}(\{v\})$ for all $v \in \mathcal{V}$, with a strict inequality for at least one element. Second, if $\left(m_{1}, \mathcal{F}_{1}\right) \sqsubset_{s}$ $\left(m_{2}, \mathcal{F}_{2}\right)$, then $\left(m_{1}, \mathcal{F}_{1}\right)$ is a specialisation both of itself and $\left(m_{2}, \mathcal{F}_{2}\right)$, hence it is in $\mathcal{M}_{12}$. Consequently, it is a solution of Equation (8).

To show that it is the unique solution inside $\mathcal{M}_{12}$, we can advocate a similar argument as in the previous proof.

This property indicates that satisfying the SCFP is also coherent with the notion of specialisation, that is the notion of inclusion that we consider as the most sensible when extending possibilistic calculus to the belief functions framework. To see that Proposition 3 do not extend to the notions of $p l$ - and $q$-inclusions, consider Example 3, the fact that one of them is either strictly $p l$ - or $q$-included in the other and that none of these two belief functions can be the solution of a conjunctive merging satisfying Eq. 6 .

The case of consonant belief structures Let $\pi_{1}, \pi_{2}$ be two possibility distributions and $\left(m_{1}, \mathcal{F}_{1}\right),\left(m_{2}, \mathcal{F}_{2}\right)$ be the corresponding consonant belief structures. In this case, it is known [15] that the minimum of distributions $\pi_{1}, \pi_{2}$ can be retrieved by a bba inside $\mathcal{M}_{m_{1} \cap m_{2}, \mathcal{V}}$ where depdendency between focal set precision is assumed. Let $0=\alpha_{0} \leq \alpha_{1} \leq \ldots \leq \alpha_{M}$ be the distinct values taken by both $\pi_{1}, \pi_{2}$ over $\mathcal{V}$, then $\min \left(\pi_{1}, \pi_{2}\right)$ correspond to the conjunctively merged belief structure $\left(m_{1 \wedge 2}, \mathcal{F}_{1 \wedge 2}\right)$ that, for $i=1, \ldots, M$, has focal sets

$$
\left\{\begin{array}{c}
E_{i}=\left(E_{i, 1} \cap E_{i, 2}\right) \\
m_{1 \wedge 2}\left(E_{i}\right)=\alpha_{i}-\alpha_{i-1}
\end{array}\right.
$$

with $E_{i, j}=\left\{v \mid \pi_{j}(v) \geq \alpha_{i}\right\}$.

This merged belief structure has a maximal cardinality, meaning that it is one of the s-least committed inside $\mathcal{M}_{m_{1} \cap m_{2}, \mathcal{V}}$ (i.e., it is among the elements of $\mathcal{M} \underset{12}{\check{L} \mathbf{s}})$. Using previous results [14,13], it is also the single least q-committed elements among $\mathcal{M}_{m_{1} \cap m_{2}, \mathcal{V}}$ (i.e., $\mathcal{M}_{12}^{\sqsubset \mathbf{q}}$ is reduced to a single element).

The next example, which completes Example 3, indicates that $\mathcal{M}$ necessarily reduced to a single element.

Example 3. Consider the two following possibility distributions $\pi_{1}, \pi_{2}$, expressed as belief structures $\left(m_{\pi_{1}}, \mathcal{F}_{\pi_{1}}\right),\left(m_{\pi_{2}}, \mathcal{F}_{\pi_{2}}\right)$

\begin{tabular}{cccc}
\multicolumn{2}{c}{$\pi_{1}=\left(m_{\pi_{1}}, \mathcal{F}_{\pi_{1}}\right)$} & \multicolumn{2}{c}{$\pi_{2}=\left(m_{\pi_{2}}, \mathcal{F}_{\pi_{2}}\right)$} \\
Focal sets & Mass & Focal sets & Mass \\
\hline$\left\{v_{0}, v_{1}, v_{2}\right\}$ & 0.5 & $\left\{v_{2}, v_{3}, v_{4}\right\}$ & 0.5 \\
$\left\{v_{0}, v_{1}, v_{2}, x_{3}, v_{4}\right\}$ & 0.5 & $\left\{v_{1}, v_{2}, v_{3}, v_{4}, v_{5}\right\}$ & 0.5
\end{tabular}


The two belief structures $\left(m_{1}, \mathcal{F}_{1}\right),\left(m_{2}, \mathcal{F}_{2}\right)$ of Example 3 can be obtained by conjunctively merging these two marginal belief structures, and none of the two belief structures in Example 3 is s-included in the other, while we do have $\left(m_{2}, \mathcal{F}_{2}\right) \sqsubset_{q}\left(m_{1}, \mathcal{F}_{1}\right)$

Note that the merged belief structure satisfying the SCFP is usually unnormalized, except when there is at least one element $v$ in $\mathcal{V}$ such that $\pi_{1}(v)=$ $\pi_{2}(v)=1$ (that is, $\left(m_{1}, \mathcal{F}_{1}\right),\left(m_{2}, \mathcal{F}_{2}\right)$ are logically consistent).

The case of probabilistic belief structures We now consider another special case where the two belief structures are equivalent to probability distributions over $\mathcal{V}$, that is when only singletons are focal sets.

Consider an arbitrary index over elements of $\mathcal{V}=\left\{v_{1}, \ldots, v_{n}\right\}$ with $|\mathcal{V}|=n$. Let $p_{1}, p_{2}$ denote two marginal probability distributions, $p_{j, i}=p_{j}\left(v_{i}\right)$ for $j=$ $1,2, i=1, \ldots, n$ and $\left(m_{1}, \mathcal{F}_{1}\right),\left(m_{2}, \mathcal{F}_{2}\right)$ their equivalent belief structures. We then have the following proposition:

Proposition 4. Let $\left(m_{1}, \mathcal{F}_{1}\right),\left(m_{2}, \mathcal{F}_{2}\right)$ be two belief structures equivalent to probability distributions $p_{1}, p_{2}$ defined on $\mathcal{V}=\left\{v_{1}, \ldots, v_{n}\right\}$. Then, the unique element $\left(m_{1 \wedge 2}, \mathcal{F}_{1 \wedge 2}\right)$ in $\mathcal{M}_{12}$ satisfying Equation (8) has, for $i=1, \ldots, n$, a joint mass

$$
m_{1 \wedge 2}\left(v_{i} \times v_{i}\right)=\min \left(p_{1, i}, p_{2, i}\right) .
$$

Proof. First, since focal sets are singletons, the only joint masses satisfying Eq. (6) which will allocate a positive mass to non-empty focal sets are of the type $m_{1 \wedge 2}\left(v_{i} \times v_{i}\right), i=1, \ldots, n$. Also note that the focal sets of any conjunctive merging will also be singletons.

Given the constraints of Eq. (6), the maximal joint mass given to $x_{i} \times x_{i}$, and therefore allocated to $x_{i}$ is $m_{1 \wedge 2}\left(x_{i} \times x_{i}\right)=\min \left(p_{1, i}, p_{2, i}\right)$. For any element $x_{i} \in \mathcal{V}$, we then have

$$
p l_{1 \wedge 2}\left(x_{i}\right)=\sum_{E \subseteq \mathcal{V}} m_{1 \wedge 2}(E)=m_{1 \wedge 2}\left(x_{i}\right)=\min \left(p_{1, i}, p_{2, i}\right) .
$$

Since we also have $p l_{j}\left(x_{i}\right)=p_{j, i}$ for $j=1,2$, this conjunctively merged belief structure, which always exists, satisfy the SCFP. It is also the only one to satisfy this principle, since any other allocation would simply give more mass to the empty set (and therefore be a strict specialisation of $\left(m_{1 \wedge 2}, \mathcal{F}_{1 \wedge 2}\right)$ ).

In this case, $\left(m_{1 \wedge 2}, \mathcal{F}_{1 \wedge 2}\right)$ is also the unique $x$-least committed element in $\mathcal{M}_{12}$, with $x \in\{p l, q, s, \pi\}$. $\left(m_{1 \wedge 2}, \mathcal{F}_{1 \wedge 2}\right)$ is also unnormalized, unless $p_{1}=p_{2}$ (in which cases SCFP enforces idempotence).

Satisfying the SCFP in general General necessary and sufficient conditions under which the merged bba has a contour function satisfying SCFP have been found by Dubois and Prade [11], however they can be difficult to check. 
Constraining the marginal belief structures to be either logically consistent (situation 1), non-conflicting (situation 2) or conflicting (situation 3), as well as requiring the conjunctively merged belief structure to be normalised are conditions that are easier to satisfy.

Let us first explore the most constraining case, that is the one where marginal belief structures are logically consistent (note that, in this case, all conjunctively merged belief structures are normalised). The next example indicates that the SCFP cannot always be satisfied, even in such a restricted case.

Example 4. Let us consider the two belief structures $\left(m_{1}, \mathcal{F}_{1}\right),\left(m_{2}, \mathcal{F}_{2}\right)$ of Example 3 as our marginal belief structures. They are logically consistent, and if there is a belief structure $\left(m_{1 \wedge 2}, \mathcal{F}_{1 \wedge 2}\right)$ in $\mathcal{M}_{12}$ that can satisfy SCFP, this belief structure should have the following contour function

$$
p l_{1 \wedge 2}\left(v_{1}\right)=0.5 \quad p l_{1 \wedge 2}\left(v_{2}\right)=1 \quad p l_{1 \wedge 2}\left(v_{3}\right)=0.5
$$

and an expected cardinality $\mathbb{C}_{\left|\left(m_{1 \wedge 2}, \mathcal{F}_{1 \wedge 2}\right)\right|}$ of 2 .

As expected cardinality is a linear function, as well as the constraints described by Eq. 6, we can easily search for the maximal expected cardinality that can attain a conjunctively merged belief structure given its marginal. The linear programming problem corresponding to our example is

$$
\max m_{1 \cap 2}\left(\left\{v_{2}\right\}\right)+2 \cdot m_{1 \cap 2}\left(\left\{v_{2}, v_{3}\right\}\right)+m_{1 \cap 2}\left(\left\{v_{2}\right\}\right)+2 \cdot m_{1 \cap 2}\left(\left\{v_{1}, v_{2}\right\}\right)
$$

under the constraints

$$
\left\{\begin{array}{c}
m_{1 \cap 2}\left(\left\{v_{2}\right\}\right)+m_{1 \cap 2}\left(\left\{v_{2}, v_{3}\right\}\right)=0.5 \\
m_{1 \cap 2}\left(\left\{v_{2}\right\}\right)+m_{1 \cap 2}\left(\left\{v_{1}, v_{2}\right\}\right)=0.5 \\
m_{1 \cap 2}\left(\left\{v_{2}\right\}\right)+m_{1 \cap 2}\left(\left\{v_{2}\right\}\right)=0.5 \\
m_{1 \cap 2}\left(\left\{v_{2}, v_{3}\right\}\right)+m_{1 \cap 2}\left(\left\{v_{1}, v_{2}\right\}\right)=0.5 \\
m_{1 \cap 2}\left(\left\{v_{2}\right\}\right)+m_{1 \cap 2}\left(\left\{v_{2}, v_{3}\right\}\right)+m_{1 \cap 2}\left(\left\{v_{2}\right\}\right)+m_{1 \cap 2}\left(\left\{v_{1}, v_{2}\right\}\right)=1
\end{array}\right.
$$

The maximal value of expected cardinality in the above problem is 1.5, and is given, for example, by $m_{1 \cap 2}\left(\left\{v_{2}, v_{3}\right\}\right)=0.5, m_{1 \cap 2}\left(\left\{v_{2}\right\}\right)=0.5$.

Since this expected cardinality is lower than the expected cardinality that should reach a conjunctively merged belief structure satisfying the SCFP. This indicates that, even when the conjunctively merged belief structure has to be normalised, the SCFP cannot be always satisfied. The two next examples indicates that the SCFP cannot always be satisfied also when the marginal random sets are non-conflicting or conflicting.

Example 5. Let us consider the space $\mathcal{V}=\left\{v_{1}, v_{2}, v_{3}\right\}$ and the two non-conflicting marginal random sets $\left(m_{1}, \mathcal{F}_{1}\right),\left(m_{2}, \mathcal{F}_{2}\right)$ summarized in the table below.

\begin{tabular}{c|ccccccc} 
Set & $\left\{v_{1}\right\}$ & $\left\{v_{2}\right\}$ & $\left\{v_{3}\right\}$ & $\left\{v_{1}, v_{2}\right\}$ & $\left\{v_{1}, v_{3}\right\}$ & $\left\{v_{2}, v_{3}\right\}$ & $X$ \\
\hline$m_{1}$ & 0.3 & 0 & 0 & 0 & 0 & 0.4 & 0.3 \\
$m_{2}$ & 0.2 & 0.1 & 0.1 & 0.2 & 0.2 & 0.1 & 0.1
\end{tabular}


Their contour functions and their minimum are summarized in the next table.

\begin{tabular}{cccc} 
Element $v_{i} \in X$ & $\pi_{1}\left(v_{i}\right)$ & $\pi_{2}\left(v_{i}\right)$ & $\min \left(\pi_{1}, \pi_{2}\right)$ \\
\hline$v_{1}$ & 0.6 & 0.7 & 0.6 \\
$v_{2}$ & 0.7 & 0.5 & 0.5 \\
$v_{3}$ & 0.7 & 0.5 & 0.5
\end{tabular}

The expected cardinality of this minimum is 1.6. However, the solution of the linear program computing the maximal expected cardinality reached by an element of $\mathcal{M}_{12}$ has the value 1.5 as solution. Therefore, there is no element in $\mathcal{M}_{12}$ satisfying the SCFP for this example.

Example 6. Let us then consider the two conflicting random sets $\left(m_{1}, \mathcal{F}_{1}\right),\left(m_{2}, \mathcal{F}_{2}\right)$ summarised below.

\begin{tabular}{cccc}
\multicolumn{2}{c}{$\left(m_{1}, \mathcal{F}_{1}\right)$} & $\left(m_{2}, \mathcal{F}_{2}\right)$ \\
Focal sets & Mass & Focal sets & Mass \\
\hline$E_{11}=\left\{v_{2}\right\}$ & 0.5 & $E_{21}=\left\{v_{1} v_{2}, v_{3}\right\}$ & 0.5 \\
$E_{12}=\left\{v_{3}\right\}$ & 0.5 & $E_{22}=\left\{v_{1}\right\}$ & 0.5
\end{tabular}

Their contour functions and their minimum are summarized in the next table.

\begin{tabular}{cccc} 
Element $v_{i} \in X$ & $\pi_{1}\left(v_{i}\right)$ & $\pi_{2}\left(v_{i}\right)$ & $\min \left(\pi_{1}, \pi_{2}\right)$ \\
\hline$v_{1}$ & 0 & 1 & 0 \\
$v_{2}$ & 0.5 & 0.5 & 0.5 \\
$v_{3}$ & 0.5 & 0.5 & 0.5
\end{tabular}

This time, the expected cardinality of the minimum is 1 , while the maximum expected cardinality reachable by an element of $\mathcal{M}_{12}$ is 0.5 (by distributing $m_{2}\left(\left\{v_{1} v_{2}, v_{3}\right\}\right)$ to either $v_{2}$ or $v_{3}$.

All these examples indicates that the SCFP, even if it extends the cautious merging of possibility distributions to belief structures, is a strong requirement difficult to satisfy in general. A possible alternative is to search for subsets of conjunctively merged belief structures satisfying a weaker contour function principle, thus working with sets of belief functions rather than with a single one. This goes in the sense of propositions made by other authors in order to deal with situations where dependencies or exact features of belief structures are not precisely known $[1,25,5]$. Such an alternative is explored in the next section.

Table 1 summarizes in which cases the SCFP can always be satisfied. It shows that, except for specific kind of belief structures, the SCFP is difficult to satisfy in general.

\subsection{Weak contour function principle (WCFP)}

In this section, we still assume that we start from marginal random sets $\left(m_{1}, \mathcal{F}_{1}\right),\left(m_{2}, \mathcal{F}_{2}\right)$ coming from sources whose dependencies is ill-known. While we still require the result of their conjunctive merging to coincide on singletons with the minimum of the contour functions $\pi_{1}, \pi_{2}$, we no longer require the result of the merging to be a single belief structure. 


\begin{tabular}{c|cccc}
\hline Situation & Constraints & Consonant Probabilistic $m_{1 \cap 2}(\emptyset)=0$ unconst. \\
\hline Logically consistent & $\sqrt{ }$ & $\sqrt{ }$ & $\times$ & $\times$ \\
Non-conflicting & $\sqrt{ }$ & $\sqrt{ }$ & $\times$ & $\times$ \\
Conflicting & $\sqrt{ }$ & $\sqrt{ }$ & N.A. & $\times$
\end{tabular}

Table 1. Satisfiability of SCFP given $\left(m_{1}, \mathcal{F}_{1}\right),\left(m_{2}, \mathcal{F}_{2}\right) \cdot \sqrt{ }$ : always satisfiable. $\times$ : not always satisfiable. N.A.: Not Applicable

Definition 9 (WCFP). Consider two belief structures $\left(m_{1}, \mathcal{F}_{1}\right),\left(m_{2}, \mathcal{F}_{2}\right)$ and $\mathcal{M}_{12}$ the set of conjunctively merged belief structures. Then, a subset $\mathcal{M} \subseteq \mathcal{M}_{12}$ is said to satisfy the weak contour function principle if, for any $v \in \mathcal{V}$,

$$
\max _{(m, \mathcal{F}) \in \mathcal{M}} \pi_{m}(v)=\min \left(\pi_{m_{1}}(v), \pi_{m_{2}}(v)\right),
$$

Any marginal random sets for which the SCFP can be satisfied also satisfy the WCFP. However, what we are searching for are subsets of $\mathcal{M}_{12}$ that always satisfy the WCFP.

Subsets of normalised merged belief functions A first subset of conjunctively merged belief structures that is interesting to explore is the one where we restrict ourselves to normalised merged belief structures (that is, all $\left(m_{\in}, \mathcal{F}_{\in}\right) \mathcal{M}_{12}$ such that $\left.m(\emptyset)=0\right)$. As the lower measure induced by this subset is equal to the lower probability of the intersection of the sets of probabilities induced by the marginal belief structures, we will denote it by $\mathcal{M}_{\mathcal{P}_{1} \cap \mathcal{P}_{2}, \mathcal{V}}$, with $\mathcal{P}_{1}$ and $\mathcal{P}_{2}$ the sets of probabilities respectively induced by $\left(m_{1}, \mathcal{F}_{1}\right)$ and $\left(m_{2}, \mathcal{F}_{2}\right)$.

Note that linear programming techniques can be used to check that a subset of merged belief functions satisfy the WCFP, as long as constraints imposed on belief structures of the subset are linear. A linear program can then be written for each $v \in \mathcal{V}$, to check whether Eq. (10) is satisfied.

As considering the subset of normalised conjunctively merged belief structure is less constraining than selecting only one of them, there will be some cases for which the SCFP cannot be satisfied, while the WCFP will be, even if we restrict ourselves to normalised belief structure. For instance, it is impossible to satisfy the SCFP for the two marginal belief structures of Example 5, while it is possible to satisfy the WCFP by restricting the subset of merged belief structures to $\mathcal{M}_{\mathcal{P}_{1} \cap \mathcal{P}_{2}, \mathcal{V}}$. The next example shows that there are cases where the WCFP cannot be satisfied when we consider the subset $\mathcal{M}_{\mathcal{P}_{1} \cap \mathcal{P}_{2}, \mathcal{V}}$.

Example 7. Consider the two marginal belief structure $\left(m_{1}, \mathcal{F}_{1}\right),\left(m_{2}, \mathcal{F}_{2}\right)$ on $\mathcal{V}=\left\{v_{1}, v_{2}, v_{3}\right\}$ such that

$$
m_{1}\left(\left\{v_{1}\right\}\right)=0.5 ; \quad m_{1}\left(\left\{v_{1}, v_{2}, v_{3}\right\}\right)=0.5,
$$


and

$$
m_{2}\left(\left\{v_{1}, v_{2}\right\}\right)=0.5 ; \quad m_{2}\left(\left\{v_{3}\right\}\right)=0.5 .
$$

The minimum of contour functions $\pi_{\min }=\min \left(\pi_{1}, \pi_{2}\right)$ is given by $\pi_{\min }\left(v_{i}\right)=0.5$ for $i=1,2,3$. The only merged bba $m_{12}$ to be in $\mathcal{M}_{\mathcal{P}_{1} \cap \mathcal{P}_{2}, \mathcal{V}}$ is

$$
m_{12}\left(\left\{v_{1}\right\}\right)=0.5 ; \quad m_{12}\left(\left\{v_{3}\right\}\right)=0.5,
$$

for which $\pi_{12}\left(v_{2}\right)=0<0.5$.

The above example is interesting, as it indicates that requiring coherence (i.e., $m(\emptyset)=0$ ) while conjunctively merging uncertain information can be, in some situation, too strong a requirement (in particular when dependencies between sources is ill-known). In particular, in the above example, the element $v_{2}$ is considered as impossible by the intersection of sets of probabilities, while both sources consider $v_{2}$ as possible.

Subsets of $s$-least committed merged belief structures Another possible solution is to consider a subset coherent with the least commitment principle. That is, given two belief structures $\left(m_{1}, \mathcal{F}_{1}\right),\left(m_{2}, \mathcal{F}_{2}\right)$, we consider the subsets $\mathcal{M} \underset{12}{\sqsubseteq \mathrm{x}}$, with $x \in\{s, p l, q, \pi\}$. Recall that

$$
\mathcal{M}_{12}^{\sqsubseteq \mathrm{x}}=\left\{(m, \mathcal{F}) \in \mathcal{M}_{12} \mid \nexists(m, \mathcal{F})^{\prime} \in \mathcal{M}_{\mathcal{P}_{1} \cap \mathcal{P}_{2}, \mathcal{V}},(m, \mathcal{F}) \sqsubset_{x}(m, \mathcal{F})^{\prime}\right\} .
$$

The following proposition shows that the subset of $s$-least committed belief structures in $\mathcal{M}_{\mathcal{P}_{1} \cap \mathcal{P}_{2}, \mathcal{V}}$ always satisfy the WCFP

Proposition 5. Let $\left(m_{1}, \mathcal{F}_{1}\right),\left(m_{2}, \mathcal{F}_{2}\right)$ be two marginal belief structure on $\mathcal{V}$. Then, the subset $\mathcal{M} \sqsubseteq \mathbf{s}$ satisfy the WCFP, in the sense that

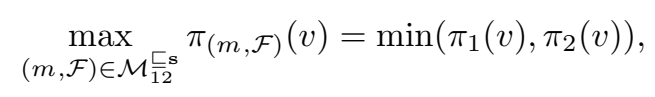

with $\pi_{1}, \pi_{2}, \pi_{(m, \mathcal{F})}$ the contour functions of, respectively, $\left(m_{1}, \mathcal{F}_{1}\right),\left(m_{2}, \mathcal{F}_{2}\right),(m, \mathcal{F})$.

Proof. To prove this proposition, we will simply show that for any $v \in \mathcal{V}$ there is at least one merged belief structure $\left(m_{v}, \mathcal{F}_{v}\right)$ in $\mathcal{M}_{12}$ such that $\pi_{\left(m_{v}, \mathcal{F}_{v}\right)}(v)=$ $\min \left(\pi_{1}(v), \pi_{2}(v)\right)$. Then, either this merged belief structure is $s$-least committed, and the problem is solved, or there is a $s$-less committed belief structure $\left(m_{x}, \mathcal{F}_{x}\right)^{\prime}$, for which

$$
\min \left(\pi_{1}(v), \pi_{2}(v)\right) \geq \pi_{\left(m_{v}, \mathcal{F}_{v}\right)^{\prime}}(v) \geq \pi_{\left(m_{v}, \mathcal{F}_{v}\right)}(v),
$$

the first inequality following from Proposition 1 and the second from the definition of $s$-inclusion.

We now have to prove that it is possible to build a merged belief structure $\left(m_{v}, \mathcal{F}_{v}\right)$ for any given $v \in \mathcal{V}$ such that $\pi_{\left(m_{v}, \mathcal{F}_{v}\right)}(v)=\min \left(\pi_{1}(v), \pi_{2}(v)\right)$. Without loss of generality, consider that, for $v, \pi_{1}(v)=\sum_{v \in E} m_{1}(E) \leq \sum_{v \in E^{\prime}} m_{2}\left(E^{\prime}\right)=$ $\pi_{2}(v)$. It is then always possible to transfer part of the masses $m_{2}\left(E^{\prime}\right), v \in E^{\prime} \in$ $\mathcal{F}_{2}$ to subsets $E \cap E^{\prime}$ containing $v$, so as to ensure $\sum_{v \in E \cap E^{\prime}} m_{v}\left(E \times E^{\prime}\right)=$ $\sum_{E \in \mathcal{F}_{v, 1}} m_{1}(E)$, while respecting Eq. (6). The same reasoning can be applied for all $v \in \mathcal{V}$ to finish the proof. 
Note that the proof of Proposition 5 also indicates that, in all cases, one can always satisfy the WCSP by choosing a set of $|\mathcal{V}|$ merged belief structures, each of them equal to the minimum of the marginal contour functions for one element $x \in \mathcal{V}$. However, how to pick them again become a question. Another interesting result follows from Proposition 5 .

Corollary 1. Let $\left(m_{1}, \mathcal{F}_{1}\right),\left(m_{2}, \mathcal{F}_{2}\right)$ be two marginal belief structure on $\mathcal{V}$. Then, the subsets $\mathcal{M} \underset{12}{\sqsubseteq \mathrm{x}}$ for $x=\{p l, q, \pi\}$ satisfy the WCFP, in the sense that

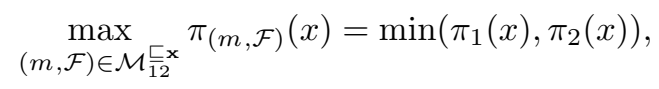

with $\pi_{1}, \pi_{2}, \pi_{(m, \mathcal{F})}$ the contour functions of, respectively, $\left(m_{1}, \mathcal{F}_{1}\right),\left(m_{2}, \mathcal{F}_{2}\right),(m, \mathcal{F})$.

Proof. Given the implications between notions of inclusions of belief structures, it is clear that, any element in $\mathcal{M} \underset{12}{\sqsubseteq \mathrm{x}}$ with $x=\{p l, q, \pi\}$ is also in $\mathcal{M} \underset{12}{\sqsubseteq \mathrm{s}}$. However, there are some elements of $\mathcal{M} \underset{12}{\sqsubseteq \mathrm{s}}$ that are not in $\mathcal{M} \underset{12}{\sqsubseteq \mathrm{x}}$. What we have to do is to show that, if one element is suppressed, then this element is of no use to satisfy Proposition 5. Let us consider two such elements $m_{1}, m_{2}$ that are in $\mathcal{M}_{12}^{\sqsubseteq \mathbf{s}}$ (i.e., they are $s$-incomparable) but are such that $m_{1} \sqsubseteq_{x} m_{2}$, hence $m_{1}$ is suppressed from $\mathcal{M}_{12}^{\sqsubseteq \mathrm{x}}$. However, for any $x \in\{p l, q, \pi\}$, we do have (see Lemma 1 )

$$
m_{1} \sqsubseteq_{x} m_{2} \Rightarrow \pi_{1} \leq \pi_{2},
$$

with $\pi_{1}, \pi_{2}$ the contour functions of $m_{1}, m_{2} . \pi_{1} \leq \pi_{2}$ ensures that $m_{1}$ is of no use when taking the maximum of all contour functions to satisfy the WCFP.

Corollary 2. Let $\left(m_{1}, \mathcal{F}_{1}\right),\left(m_{2}, \mathcal{F}_{2}\right)$ be two marginal belief structure on $\mathcal{V}$. Then, if any of the subsets $\mathcal{M} \stackrel{\square}{\square} \mathbf{x}$ with $x=\{s, p l, q, \pi\}$ is reduced to a singleton $\left(m_{x}, \mathcal{F}_{x}\right)$, then this element satisfy the SCFP.

This is, for instance, the case with $\mathcal{M} \underset{12}{\sqsubseteq \mathbf{q}}$ when both $\left(m_{1}, \mathcal{F}_{1}\right),\left(m_{2}, \mathcal{F}_{2}\right)$ are consonant. As for SCFP, Table 2 summarises for which subset of merged belief

\begin{tabular}{|c|c|c|c|c|c|}
\hline Situation $\quad$ Subset & $\mathcal{M}_{\mathcal{P}_{1} \cap \mathcal{P}_{2}}$ & $\mathcal{M} \frac{\sqsubseteq \mathrm{s}}{12}$ & $\mathcal{M} \frac{\sqsubseteq p}{12}$ & $\mathcal{M} \underset{12}{\check{q}}$ & $\mathcal{M} \sqsubseteq \pi$ \\
\hline Logically consistent & $\sqrt{ }$ & $\sqrt{ }$ & $\sqrt{ }$ & $\sqrt{ }$ & $\sqrt{ }$ \\
\hline Non-conflicting & $\times$ & $\sqrt{ }$ & $\sqrt{ }$ & $\sqrt{ }$ & $\sqrt{ }$ \\
\hline Conflicting & N.A. & $\sqrt{ }$ & $\sqrt{ }$ & $\sqrt{ }$ & $\sqrt{ }$ \\
\hline
\end{tabular}
structure the WCFP is always satisfiable.

Table 2. Satisfiability of WCFP given $\left(m_{1}, \mathcal{F}_{1}\right),\left(m_{2}, \mathcal{F}_{2}\right) \cdot \sqrt{ }$ : always satisfiable. $\times$ : not satisfiable in general. N.A.: Not Applicable 


\section{Conclusions}

In this paper, we have studied to what extent the idempotent rule used in possibility theory to cautiously merge possibility distribution can be extended to the more general framework of belief structures. In order to achieve such an extension, we have proposed two principles, respectively the strong and weak contour function principle. These principles require the contour function of merged belief structures to be equal to the minimum of the marginal contour functions.

Our results indicates that the strong version of this principle (selecting only one merged belief structure) cannot be always satisfied, but that it is relatively easy to satisfy it if we consider the weaker version of this principle, which allows the result of the merging to be a set of belief structures. In this latter case, restricting to least-committed merged belief structures appears to be a good solution, and is coherent with the least-commitment principle.

From a practical standpoint, our results are rather negative, as they do not lead to a cautious merging rule extending the idempotent possibilistic rule easy to use. Thus, they do not allow to cope easily with ill-known dependencies between information sources.

Our results have a theoretical interest, as they tends to confirm that the use of sets of belief structures rather than of single belief structure is sometimes desirable, particularly when dependencies are ill-known. This is in agreement with similar treatments done with precise probabilities when dependencies between variables are not known [25]. This indicates that belief functions alone are perhaps not always sufficient to treat some problems.

Section 3.2 also indicates that restricting ourselves to normalised merged belief functions is also too constraining if we want to comply with our principles. This indicates that requiring coherence is also perhaps too strong a requirement in some situations. This is in agreement with the Transferable Belief Model [23] and the open world assumption, where unnormalized belief structures are authorised.

Another question that remains to be solved is to know if, given two marginal belief structures, considering the top elements of the conjunctively merged belief structures with respect to the complete ordering induced by expected cardinality allows to satisfy the WCFP? that is, if only the conjunctively merged belief structures with maximal expected cardinality are considered, do they satisfy the WCSP. Replying to this question would be interesting, since if the answer is affirmative, this would give a practical way to extract a subset of conjunctively merged belief functions satisfying the WCSP, by using linear programming techniques to extract all those belief functions having maximal expected cardinality.

\section{References}

1. Th. Augustin. Generalized basic probability assignments. I. J. of General Systems, 34(4):451-463, 2005. 
2. M. Cattaneo. Combining belief functions issued from dependent sources. In Proc. Third International Symposium on Imprecise Probabilities and Their Application (ISIPTA'03), pages 133-147, Lugano, Switzerland, 2003.

3. Alain Chateauneuf. Combination of compatible belief functions and relation of specificity. In Advances in the Dempster-Shafer theory of evidence, pages 97-114. John Wiley \& Sons, Inc, New York, NY, USA, 1994.

4. A.P. Dempster. Upper and lower probabilities induced by a multivalued mapping. Annals of Mathematical Statistics, 38:325-339, 1967.

5. T. Denoeux. Reasoning with imprecise belief structures. I. J. of Approximate Reasoning, 20:79-111, 1999.

6. T. Denoeux. Conjunctive and disjunctive combination of belief functions induced by non-distinct bodies of evidence. Artificial Intelligence, 172:234-264, 2008.

7. S. Destercke, D. Dubois, and E. Chojnacki. Cautious conjunctive merging of belief functions. In Proc. Eur. Conf. on Symbolic and Quantitative Approaches to Reasoning with Uncertainty, pages 332-343, 2007.

8. D. Dubois and H. Prade. On the unicity of the dempster rule of combination. International Journal of Intelligent Systems, 1:133-142, 1986.

9. D. Dubois and H. Prade. A set-theoretic view on belief functions: logical operations and approximations by fuzzy sets. I. J. of General Systems, 12:193-226, 1986.

10. D. Dubois and H. Prade. Possibility Theory: An Approach to Computerized Processing of Uncertainty. Plenum Press, New York, 1988.

11. D. Dubois and H. Prade. Fuzzy sets, probability and measurement. European Journal of Operational Research, 40:135-154, 1989.

12. D. Dubois and H. Prade. Consonant approximations of belief functions. I.J. of Approximate reasoning, 4:419-449, 1990.

13. D. Dubois, H. Prade, and P. Smets. New semantics for quantitative possibility theory. In G. de Cooman, T. Fine, and T. Seidenfeld, editors, ISIPTA'01, Proceedings of the Second International Symposium on Imprecise Probabilities and Their Applications. Shaker Publishing, 2001.

14. D. Dubois, H. Prade, and P. Smets. A definition of subjective possibility. I. J. of Approximate Reasoning., 48:352-364, 2008.

15. D. Dubois and R.R. Yager. Fuzzy set connectives as combination of belief structures. Information Sciences, 66:245-275, 1992.

16. E.P. Klement, R. Mesiar, and E. Pap. Triangular Norms. Kluwer Academic Publisher, Dordrecht, 2000.

17. G.J. Klir. Uncertainty and information measures for imprecise probabilities : An overview. In Proc. 1st International Symposium on Imprecise Probabilities and Their Applications, Ghent, Belgium, 1999.

18. S. Moral and J. Sagrado. Aggregation of imprecise probabilities. In B. BouchonMeunier, editor, Aggregation and Fusion of Imperfect Information, pages 162-188. Physica-Verlag, Heidelberg, 1997.

19. G. Shafer. A Mathematical Theory of Evidence. Princeton University Press, New Jersey, 1976.

20. P. Smets. Combining nondistinct evidences. In Int. Conf. of the North American Fuzzy Information Processing Society (NAFIPS'86), pages 544-549, 1986.

21. P. Smets. The canonical decomposition of a weighted belief. In Proc. Int. Joint. Conf. on Artificial Intelligence, pages 1896-1901, Montreal, 1995.

22. P. Smets. Analyzing the combination of conflicting belief functions. Information Fusion, 8:387-412, 2006.

23. P. Smets and R. Kennes. The transferable belief model. Artificial Intelligence, 66:191-234, 1994. 
24. P. Walley. Statistical reasoning with imprecise Probabilities. Chapman and Hall, New York, 1991.

25. R.C. Williamson and T. Downs. Probabilistic arithmetic i : Numerical methods for calculating convolutions and dependency bounds. I. J. of Approximate Reasoning, 4:8-158, 1990.

26. L.A. Zadeh. Fuzzy sets as a basis for a theory of possibility. Fuzzy Sets and Systems, 1:3-28, 1978. 